\title{
Diel changes in adipokinetic response and walking activity of Pyrrhocoris apterus (Heteroptera) in relation to physiological status and wing dimorphism
}

\author{
ANNA MAXOVÁ ${ }^{1,2}$, DALIBOR KODRÍK $^{1^{*}, 2}$, Rostislav ZEMEK ${ }^{1}$ and RADOMíR SOCHA ${ }^{1}$ \\ ${ }^{1}$ Institute of Entomology, Academy of Sciences, Branišovská 31, České Budějovice, 370 05, Czech Republic \\ ${ }^{2}$ Faculty of Biological Sciences, University of South Bohemia, Branišovská 31, České Budějovice, 370 05, Czech Republic
}

Key words. Pyrrhocoris apterus, Pya-AKH, adipokinetic response, walking activity, diurnal rhythm, diapause, wing polymorphism

\begin{abstract}
Three experimental groups of adult females of Pyrrhocoris apterus (reproductive brachypterous, diapausing brachypterous, and macropterous with non-diapause type of reproductive arrest) were analysed for their dose dependency of adipokinetic response, sensitivity of the fat body to Pya-AKH and locomotor activity during a 24 hour cycle. The greatest adipokinetic response (lipid elevation after hormonal treatment) was observed in macropterous, lower in diapausing brachypterous and the lowest in reproductive brachypterous females. Macropterous females also exhibited the highest activity of walking, while the lowest locomotor activity was found in diapausing brachypters. Diel changes of adipokinetic response were recorded in all three experimental groups, with maximal response occurring during the day at 6-7 h after the onset of light. On the contrary, the minimal adipokinetic response was found in the night and early after the onset of light. No significant differences in diel rhythms were observed between groups exposed to various day lengths. Diel changes in locomotor activity were found only in reproductive brachypterous females and females of macropterous morph, and showed a diurnal rhythm with only one peak of activity during a 24 hour cycle. In the latter, the rhythmic pattern of walking activity was positively correlated with diel changes of adipokinetic response. This indicates an important role of Pya-AKH in dispersal strategy of macropterous females of this heteropteran.
\end{abstract}

\section{INTRODUCTION}

Adipokinetic hormone $(\mathrm{AKH})$ is one of the best known neuropeptides that controls and regulates energy metabolism in insects. Many AKHs have been isolated from corpora cardiaca and characterized in a great number of insect orders (Gäde et al., 1997; Gäde, 1999; Lorenz et al., 1999), and recently also in the firebug Pyrrhocoris apterus, a representative species of the suborder Heteroptera (Kodrík et al., 2000). Almost all of these hormones are octa-, nona- or decapeptides with characteristic structural similarities (Gäde et al., 1997), and they are grouped into the $\mathrm{AKH} / \mathrm{RPCH}$ (Adipokinetic Hormone/Red Pigment Concentrating Hormone) family. The first reported effect of AKHs was the lipid mobilization from the fat body of Locusta migratoria, which is known to provide an energy fuel for long term flight (Mayer \& Candy, 1969). AKHs generally play an important role in stress situations, with the need for activation of energy stores. The stimulation of walking activity by $\mathrm{AKH}$ was recently proven in P. apterus (Socha et al., 1999a, b; Kodrík et al., 2000).

$P$. apterus is a flightless species producing two major morphs, macropterous (long-winged) and brachypterous (short-winged). They differ, in addition to the length of the pre-oviposition period, in other physiological, biochemical and behavioural parameters; e.g., the quantity of hexameric storage protein in haemolymph (Šula et al., 1995; Socha \& Šula, 1996), feeding behaviour, digestive physiology, and glycogen and lipid content in the fat body (Socha et al., 1997, 1998). The differences in the life strategy of these morphs may be related to the flight capability of their macropterous ancestors. This presumption is supported by the finding that, in spite of the loss of flight capability during evolution, the recent representatives of the macropterous morph of $P$. apterus have retained wing flapping capability (Socha \& Zemek, 2000a). Recent studies showed that the macropterous morph also differs from the brachypterous one by a more intensive response to AKH treatment (Kodrík \& Socha, 1999; Socha \& Kodrík, 1999) and by higher activity of walking (Socha \& Zemek, 2000b). This finding suggests that AKH plays an important role in mobilization of lipid reserves from the fat body in the macropterous morph to energetically ensure their enhanced walking activity and higher tendency of dispersal.

Recently, data on physiological and behavioural differences between reproductive and diapausing adults of $P$. apterus have been reported, for example differences in the amount of hexameric storage protein in the haemolymph (Šula et al., 1995), adipokinetic response (Socha \& Kodrík, 1999) and activity of walking (Socha \& Zemek, 2000b). However, despite intensive research, our knowledge of the relationships between diapause, lipid mobilization and locomotor activity is still insufficient.

A number of physiological and behavioural functions in insects related to certain concentrations of biomolecules are known to show rhythmic diurnal changes (Saunders, 1982). Rhythms in the function of CNS and endocrine systems appear to play an important role in the control of various physiological and behavioural rhythms. $\mathrm{AKH}$, as mentioned above, controls the release of lipids

\footnotetext{
* Corresponding author: e-mail:kodrik@entu.cas.cz, fax: ++420-38-43625
} 
from the fat body into the haemolymph. The insect haemolymph has a relatively high total lipid content which varies under different factors such as developmental stage, starvation, disease, locomotion (Mullins, 1985), diapause (Socha \& Kodrík, 1999) and photoperiodicity (Das et al., 1993). The diel changes of AKH content in corpora cardiaca and in adipokinetic response has only been reported for the cricket Acheta domesticus (Das et al., 1993). However, data on diel changes in adipokinetic response and locomotor activity, and their relationship to wing polymorphism of insects are missing.

The main aim of this study was therefore to test the hypotheses that (1) adipokinetic response and locomotion of $P$. apterus exhibit diel rhythms, (2) these rhythms correlate in time, and (3) the dose-dependent adipokinetic response and diel rhythms in adipokinetic response and walking activity are related to the wing dimorphism and physiological status of the bugs. The results obtained will extend our knowledge about chronobiology of behavioural and physiological functions in this heteropteran species.

\section{MATERIALS AND METHODS}

Experimental animals

Two stock cultures of $P$. apterus, originating from the wild population collected at České Budějovice (Czech Republic, $49^{\circ} \mathrm{N}$ ), were used in the present study (Socha \& Šula, 1996). All stages from egg to adult were kept in small glass jars with linden seeds and water ad libitum at constant temperature of 26 $\pm 1^{\circ} \mathrm{C}$. Three experimental groups of adult females were studied: reproductive brachypterous, diapausing brachypterous and macropterous. Reproductive brachypterous females and macropterous females with reproductive arrest of non-diapause type were reared under long-day conditions $(18 \mathrm{~L}: 6 \mathrm{D} \mathrm{h})$. Diapausing brachypterous females were maintained under short-days conditions $(12 \mathrm{~L}: 12 \mathrm{D} \mathrm{h})$. For analyses of diel rhythms in adipokinetic response and walking activity, 10-day-old adult females of all experimental groups were used (see Socha \& Kodrík, 1999).

\section{Hormonal treatment}

The Pya-AKH from P. apterus (Kodrík et al., 2000) was used in our experiments. A stock solution of the hormone was prepared in 20\% methanol in Ringer saline and the working dilutions were prepared to give the desired amount of the peptide per $2 \mu \mathrm{l}$ solution. This volume was injected by a syringe $(10 \mu 1$, Hamilton Co., Reno, Nevada) through the metathoracicabdominal intersegmental membrane to the thorax of each experimental female used in the determination of adipokinetic response. A dose of $10 \mathrm{pmol}$ of the hormone was used for analysis of diel changes of adipokinetic response, because it showed the maximal hyperlipaemic effect in the bug (see Results). Control females were injected with $2 \mu$ of $20 \%$ methanol in Ringer saline in the same way.

\section{Haemolymph sampling and lipid mobilization bioassay}

The haemolymph samples were collected before and $90 \mathrm{~min}$ after the hormonal treatment. The top of antenna was cut off by scissors and a drop of haemolymph was leaked onto a piece of parafilm M. An aliquot of $0.5 \mu 1$ haemolymph was taken by a micropipette from the drop and used for the determination of lipids. Lipid concentration in the haemolymph samples was determined by a method based on the supho-phosphovanillin test (Zöllner \& Kirsch, 1962; modified by Holwerda et al., 1977 and Van Marrewijk et al., 1984). The optical densities, recorded at $546 \mathrm{~nm}$ in a spectrophotometer (UV-1601 Shimadzu), were converted to $\mathrm{mg}$ lipids per $\mathrm{ml}$ of haemolymph with the aid of a calibration graph, based on known aliquots of oleic acid, using MS Excel. Results were expressed as a mean \pm SD $(n=7-14)$ of haemolymph lipid elevation (difference of lipid levels after and before injection).

Monitoring of locomotor activity and data acquisition

Adult females destined for investigation of diel changes of locomotor activity were individually placed into one of four compartments $(4.5 \times 6.5 \mathrm{~cm})$ of a translucent plastic tray. Measurement of activity was carried out in a climate-controlled cabinet under the same photoperiodic conditions used during their development. The observation arena was scanned using a high resolution black and white CCD video camera. The locomotor activity was measured by means of a computer image analysis system where the motion was detected by comparison of two subsequent images. The software allowed separate analysis of four regions of image, thus providing simultaneous measurement of bug activity in each of the four compartments in the observation arena. Scores of all samples for each bug were integrated over a 1-hour period and the total time spent in movement on the 10th day after adult ecdysis was calculated. For detailed description of experimental design and data acquisition see Socha et al. (1997) and Socha \& Zemek (2000b).

Data presentation and statistical analyses

The results of adipokinetic responses and locomotor activities were plotted and the curves fitted by means of the graphic program Prism (GraphPad Software, version 3.0, San Diego, CA, USA). An unpaired t-test with the help of the program Instat 1.15 (GraphPad Software, San Diego, USA) was used for statistical analyses of the dose-response effect of Pya-AKH. Two way ANOVA $(\alpha=0.05)$ was used for statistical analysis of diel changes of lipid mobilization in all experimental groups of insects and ANOVA for repeated measurement $(\alpha=0.05)$ of diel changes in locomotor activity to estimate the effect of morphs, diapause and length of photoperiod on the rhythmicity of these variables.

\section{RESULTS}

The analysis of the dose-response effect of Pya-AKH on 10-day-old reproductive and diapausing brachypterous females and females of macropterous morph revealed that haemolymph lipid elevations were dose-dependent in all experimental groups (Fig. 1). The intensity of the adipokinetic response in all three experimental groups increased with higher doses and was the highest for the dose of 10 pmol Pya-AKH. The doses 20-40 pmol were less active. The highest intensity of adipokinetic response was recorded in macropters, and the lowest one in the reproductive brachypterous females. A comparison of the obtained data showed significant differences of adipokinetic responses between macropterous and reproductive brachypterous females for doses of $0.5-10 \mathrm{pmol}$, and between both groups of brachypters (reproductive and diapausing) for doses of $0.1,0.5$ and 5 pmol $(P<0.05)$. No dramatic differences were recorded when the adipokinetic response of the females with different modes of inhibition of reproduction (i.e. macropters with nondiapause type of reproductive arrest and diapausing brachypters) were compared - significant differences were recorded only for the doses of 0.1 and 10 pmol of the hormone. Values of adipokinetic responses differed signifi- 


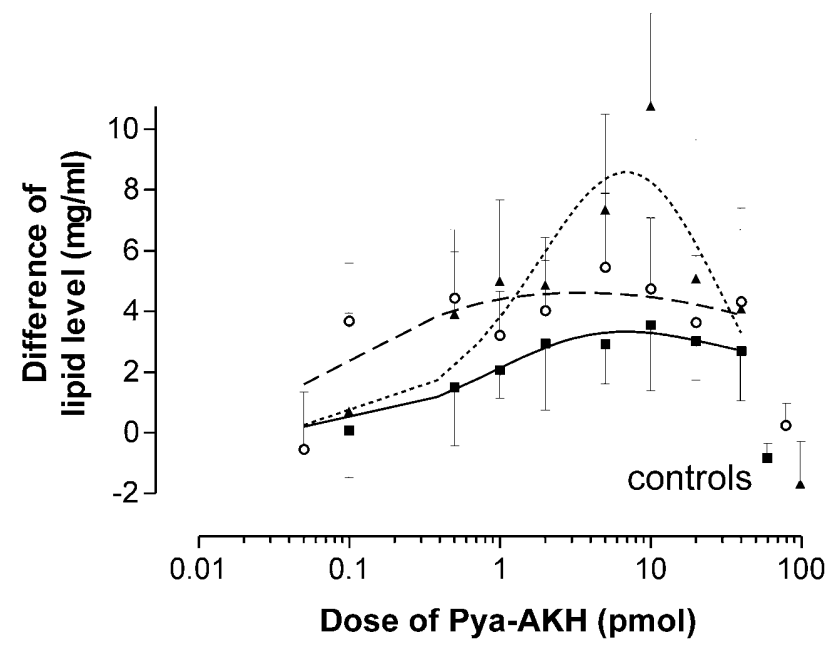

Fig. 1. Effect of increasing doses of Pya-AKH on the elevation of haemolymph lipid level in 10-day-old females of $P$. apterus. Reproductive brachypterous $(\mathbf{\square},-)$, diapausing brachypterous $(\mathrm{O},---)$ and macropterous $(\boldsymbol{\Lambda},-------)$ females. Each point represents the mean $\pm \mathrm{SD}(n=7-14)$. Control bugs were injected with $20 \%$ methanol in Ringer saline.

cantly from the corresponding controls (injection of $20 \%$ methanol in saline) $(P<0.05)$ for doses of $0.5-40 \mathrm{pmol}$ Pya-AKH in the reproductive brachypterous and macropterous females, and for doses of $0.1-40 \mathrm{pmol}$ in the diapausing brachypterous ones.

All three groups of females analysed showed significant changes in lipid mobilization by AKH injected during a 24 hours period (Fig. 2). The time-dependent course of adipokinetic responses displayed a similar diurnal pattern, with the highest responsiveness to $\mathrm{AKH}$ at 6-7 h after the onset of light and the lowest one in scotophase. The effect of time on adipokinetic response was not significant at $\alpha=0.05$, whereas the experimental group affected the adipokinetic response (two way ANOVA, $P<0.0001$ ).

Rhythmic diurnal patterns in locomotion, with significantly higher activity in the middle of the photophase and very low activity in the scotophase, were found in macropterous and reproductive brachypterous females. No rhythmic pattern was found in diapausing brachypterous bugs, because of their very low activity (Fig. 3). Statistical analysis revealed a highly significant effect of all studied phenomena (both time and experimental groups) on locomotor activity pattern (ANOVA for repeated measurement, $P<0.0001$ ).

To elucidate the relationship between mobilization of lipid reserves from the fat body and the locomotion of $P$. apterus, correlations between temporal courses of adipokinetic response and locomotor activity were made for all experimental groups of females. A positive correlation between both rhythms was demonstrated only in females of macropterous morph $\left(\mathrm{r}^{2}=0.62, P=0.0023\right)$ (Fig. 4).

\section{DISCUSSION}

Insects, like other organisms, usually restrict their activity to certain times of the diel cycle (Saunders,

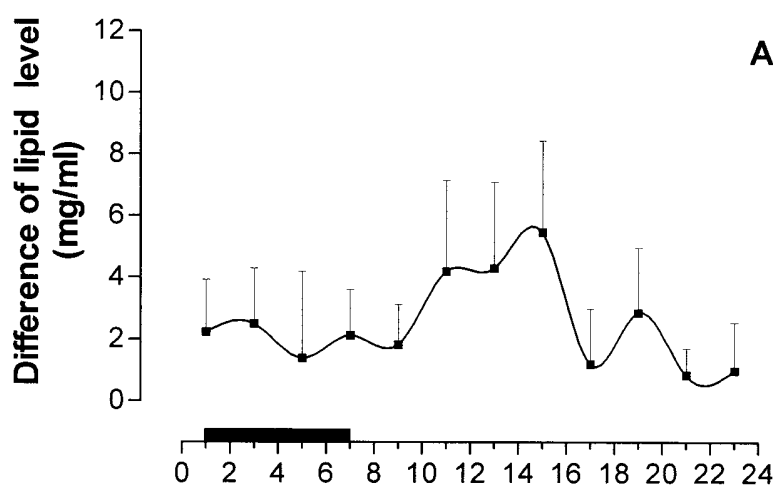

Time (hours)

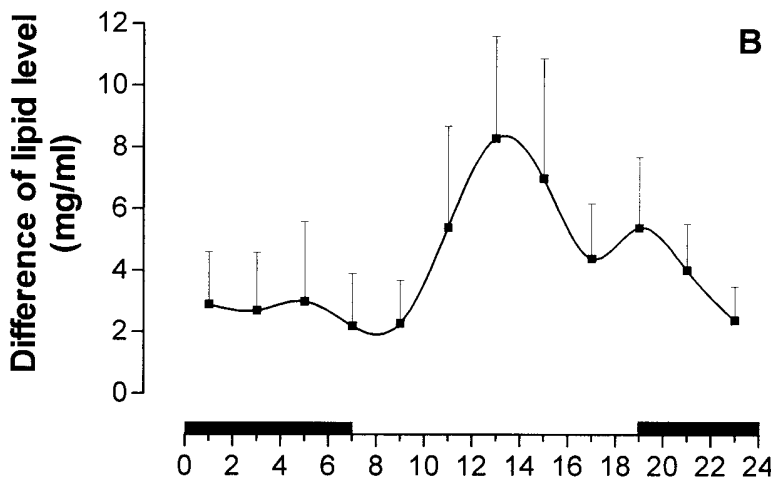

Time (hours)

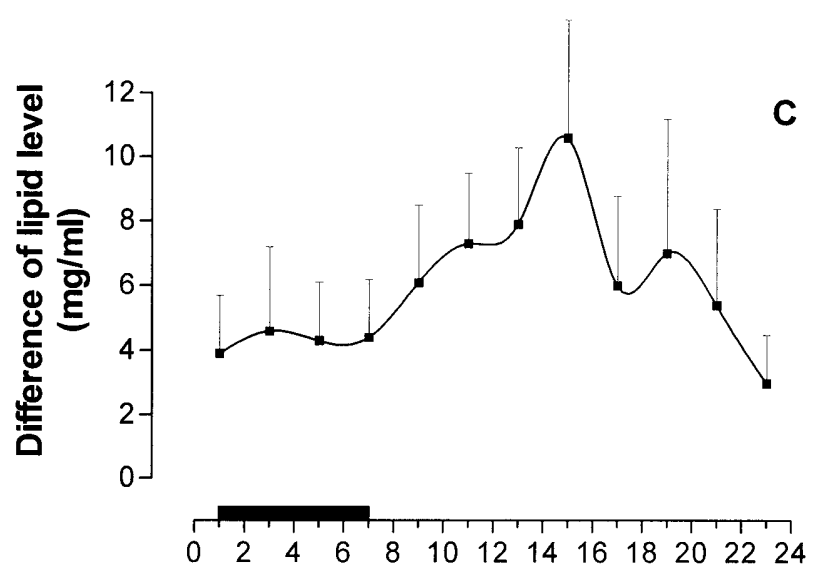

Time (hours)

Fig. 2. Diel changes of the adipokinetic response in 10day-old females of $P$. apterus. A - reproductive brachypterous; $\mathrm{B}$ - diapausing brachypterous; $\mathrm{C}$ - macropterous females. Each point (expressed as difference of lipid levels after and before injection of 10 pmol Pya-AKH) represents the mean $\pm \mathrm{SD}(n=$ $7-14)$. The adipokinetic response in the bugs treated with solvent only was found to be very low at any time of day (data not shown). The scotophase is represented by the solid bar in the $\mathrm{x}$-axis. 


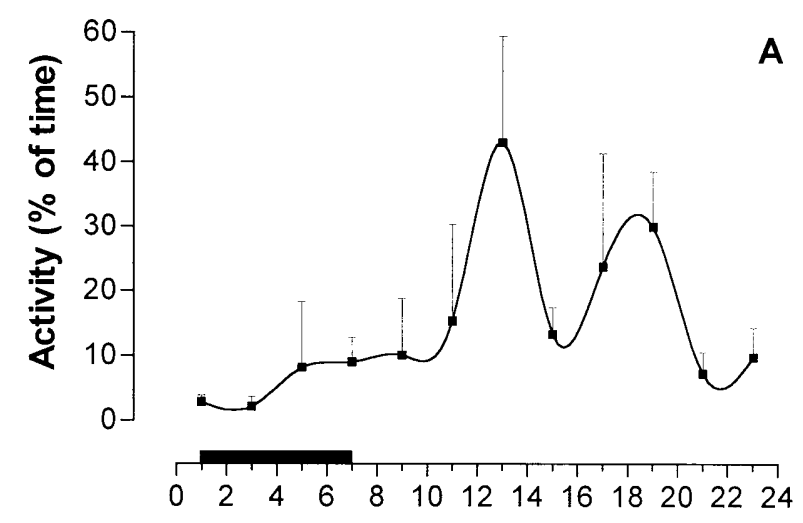

Time (hours)

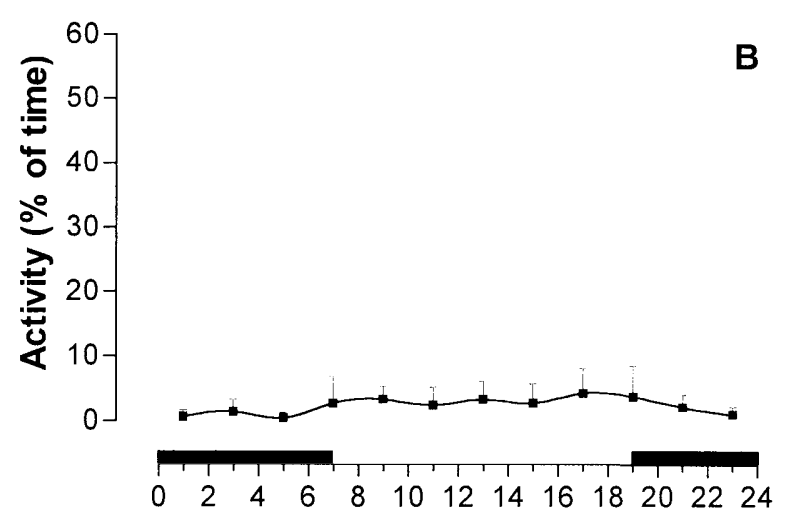

\section{Time (hours)}

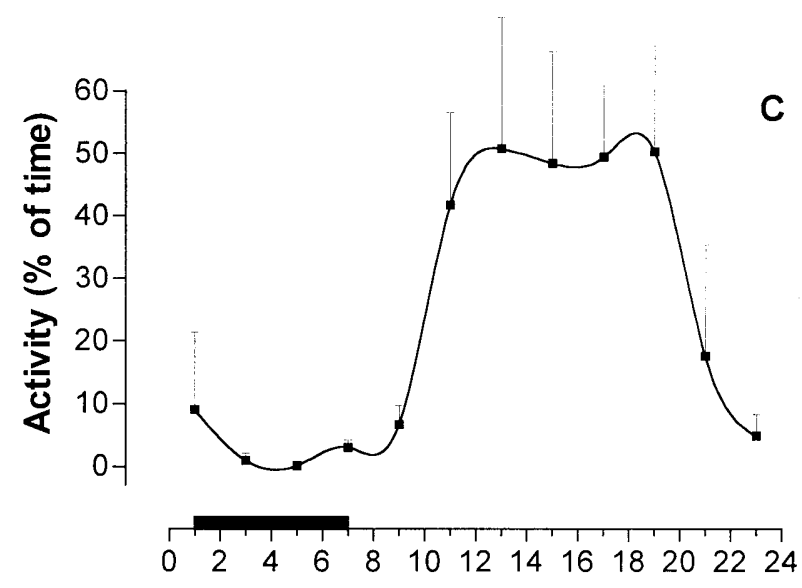

Time (hours)

Fig. 3. Diel changes of locomotor activity in 10-day-old females of $P$. apterus. A - reproductive brachypterous; B - diapausing brachypterous; $\mathrm{C}$ - macropterous females. Each point represents the mean $\pm \mathrm{SD}$ of $\%$ time spent by walking $(n=4)$. The scotophase is represented by the solid bar in the $\mathrm{x}$-axis.

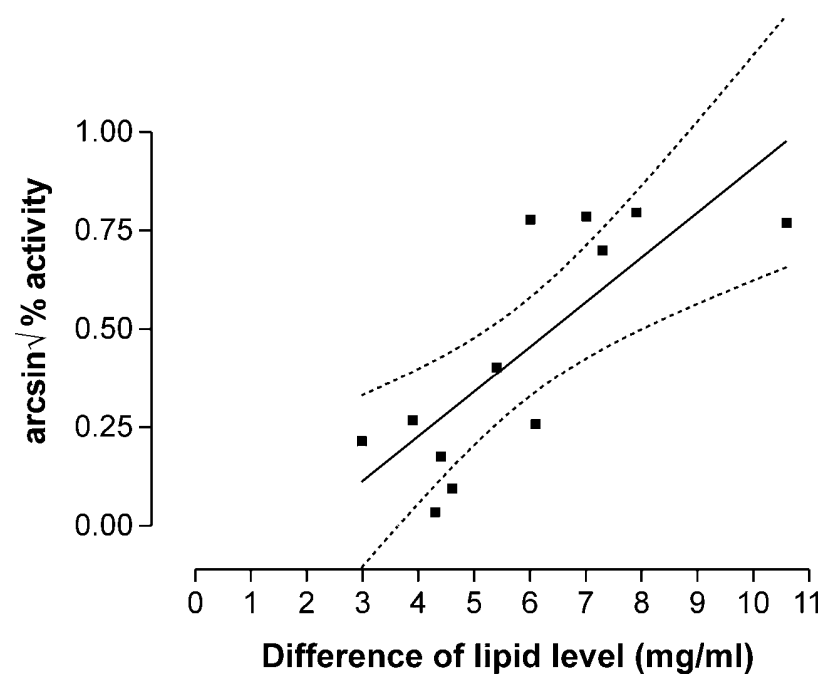

Fig. 4. Relationship between diurnal rhythms of lipid mobilization by Pya-AKH and locomotor activity in 10-day-old macropterous females of $P$. apterus. Solid line: regression line $\left(\mathrm{r}^{2}=\right.$ $0.62, P=0.0023$ ), dashed lines: $95 \%$ confidence intervals.

1982). In the present study, we demonstrated that the adipokinetic response in $P$. apterus is characterized by a diurnal rhythm. Rhythmicity of adipokinetic response was first demonstrated in the house cricket $A$. domesticus by Das and his co-workers (1993), who found that the major peaks of fat body sensitivity of this species to injection of AKH was correlated with two major peaks in the levels of haemolymph lipids. Because $A$. domesticus is a nocturnal insect, the maximum activity was observed during scotophase. In $P$. apterus, the maximum intensity of adipokinetic response occurred during the photophase, and was the highest in macropterous and the lowest in reproductive brachypterous females. These data are in accordance with our previous results and the knowledge about the effect of AKH in this bug: the adipokinetic response is related to the wing dimorphism and physiological status of $P$. apterus, as has been reported previously, when adipokinetic hormone I from Locusta migratoria (LomAKH-I) was used (Kodrík \& Socha, 1999; Socha \& Kodrík, 1999).

We found that $P$. apterus exhibits diel changes in locomotion, with the maximum of walking activity occurring during photophase. Similar results were also obtained when the walking activity of the bugs was measured during the first 14 days of their adult life; they moved predominantly during light phase and were inactive during scotophase (Socha \& Zemek, 2000b). The greatest locomotor activity during the light period was demonstrated also for another pyrrhocorid Dysdercus intermedius (Schmidt et al., 1992). A multiple comparison test of all groups of $P$. apterus females studied revealed differences between their diurnal locomotor patterns. The highest walking activity during photophase was observed in macropterous and the lowest in diapausing brachypterous females. The obviously very low activity in diapausing brachypterous females could be related to the lower activity of metabolism and accumula- 
tion of energy reserves for hibernation. The situation is more complicated in reproductive brachypterous females. The rhythms of the locomotor activity appear to be synchronized with the reproductive cycle and the feeding activity (Socha et al., 1997). During oviposition (on days 6 and 10 after adult ecdysis) the locomotor and feeding activities of females decreased. However, there are many individual differences between reproductive ovipositing females in time. Synchronization of daily patterns of locomotor and feeding activities has also been reported for the German cockroach Blattella germanica (Lee \& Wu, 1994). In P. apterus, a considerable diurnal rhythm in locomotor activity was shown to occur in 10-day-old macropterous females, which are characterized by reproductive arrest of a non-diapause type, fasting, high lipid concentration in the haemolymph, and related high adipokinetic response (Socha et al., 1998; Kodrík \& Socha, 1999; Socha \& Kodrík, 1999). The different rates of lipid mobilization in females of two wing morphs of $P$. apterus is probably related to their different locomotor and dispersal activities. This hypothesis is supported by recent findings that locomotor activity of macropterous females is higher than that of brachypterous females (Socha \& Zemek, 2000b), and that the locomotor activity of $P$. apterus is stimulated by both Lom-AKH-I and Pya-AKH (Socha et al., 1999a, b; Kodrík et al., 2000).

The diel rhythm of locomotor activity in macropterous females of $P$. apterus is positively correlated with the effect of Pya-AKH on lipid mobilization. This is the first report of this phenomenon in insects. The locomotor activity of $A$. domesticus was found to be correlated with the diel changes in the content of neurosecretory cells in the brain (Cymborowski, 1983). In our present study, the peak of locomotor activity in P. apterus females was correlated with the peak of sensitivity of their fat bodies to Pya-AKH. Positive correlation was found also between the temporal course of the stimulatory effect of LomAKH-I on locomotion and its effect on lipid mobilization (Socha et al., 1999b). These findings are in accordance with the metabolic pathway of a hypothetical model describing the possible mechanism of AKH stimulatory effect on locomotion via mobilization of lipids (Socha et al., 1999a). Having not tested the haemolymph lipid levels, AKH levels and sensitivity to the hormone in $P$. apterus females maintained under constant darkness, we can say nothing concerning the endogenous aspects of these rhythms.

In summary, this paper demonstrates for the first time, an occurrence of diel changes in adipokinetic response in $P$. apterus, confirms that these changes are related to wing polymorphism and diapause, and shows the positive correlation between rhythms in adipokinetic response and locomotor activity for only females of macropterous morph.

ACKNOWLEDGEMENTS. This study was supported by the Institute of Entomology project No. 5007907 (Academy of Sciences of the Czech Republic), partially by grant Kontakt CZE- 00-004 (ME-417) from the Ministry of Education of the Czech Republic (DK) and partially by grant No. 206/99/0068 from the
Grant Agency of the Czech Republic (RS). The authors thank D. Rienesslová for her technical assistance.

\section{REFERENCES}

Cymborowski B. 1983: Daily changes in synthesis and accumulation of neurosecretion in the brain of house cricket. $J$. Int. Cycle Res. 14: 111-116.

Das S., Meier O.W. \& Woodring J. 1993: Diel rhythms of adipokinetic hormone, fat body response and haemolymph lipid and sugar levels in the house cricket. Physiol. Entomol. 18: 233-239.

GÄDE G. 1999: Presence of an adipokinetic peptide in the corpus cardiacum of Dermaptera but not in the neurohaemal aorta, and chemical and functional identification of the peptide. Physiol. Entomol. 24: 327-332.

GÄde G., Hoffmann K.H. \& SpRING J.H. 1997: Hormonal regulation in insects: facts, gaps, and future directions. Physiol. Rev. 77: 963-1032.

Holwerda D.A., Van Doorn J. \& Beenakkers A.M.T. 1977: Characterization of the adipokinetic and hypertrehalosaemic substances from the locust corpus cardiacum. Insect Biochem. 7: 151-157.

Kodrík D. \& Socha R. 1999: A Mediterranean population of Pyrrhocoris apterus (Heteroptera: Pyrrhocoridae) exhibits wing morph-related differences in adipokinetic response. Eur. J. Entomol. 96: 327-330.

Kodrík D., Socha R., ŠImeK P., Zemek R. \& Goldsworthy G.J. 2000: A novel member of the AKH/RPCH family that stimulates locomotor activity in the firebug, Pyrrhocoris apterus (Heteroptera). Insect Biochem. Mol. Biol. 30: 489-498.

LeE H.-J. \& Wu Y.-L. 1994: Mating effect on the feeding and locomotion of the German cockroach, Blattella germanica. Physiol. Entomol. 19: 39-45.

Lorenz M.W., Roland K., Woodring J., Hoffmann K.H. \& GäDE G. 1999: Hypertrehalosaemic peptides in the honeybee (Apis mellifera): purification, identification and function. $J$. Insect Physiol. 45: 647-653.

MAYER R.J. \& CANDY D.J. 1969: Control of haemolymph lipid concentration during locust flight: an adipokinetic hormone from the corpora cardiaca. J. Insect Physiol. 15: 611-620.

Mullins D.E. 1985: Chemistry and physiology of the haemolymph. In Kerkut G.A. \& Gilbert L.I. (eds): Comprehensive Insect Physiology Biochemistry and Pharmacology, Vol. 3. Pergamon Press, Oxford, pp. 355-400.

SAUnders D.S. 1982: Insect Clocks. 2nd ed. Pergamon Press, New York, $279 \mathrm{pp}$

Schmidt G.H., Scheer A. \& Melber A. 1992: Untersuchungen zur Tagesrhytmik im Lauf- und Nahrungssuchverhalten bei Baumwollwanzen (Dysdercus intermedius Dist.) (Insecta: Heteroptera). Zool. Jb. Physiol. 96: 481-509.

SOCHA R. \& KoDrík D. 1999: Differences in adipokinetic response of Pyrrhocoris apterus (Heteroptera) in relation to wing dimorphism and diapause. Physiol. Entomol. 24: 278284.

Socha R. \& Šula J. 1996: Differences in haemolymph proteins in relation to diapause and wing dimorphism in Pyrrhocoris apterus (Heteroptera: Pyrrhocoridae). J. Comp. Physiol. 166B: $382-376$.

SOCHA R. \& ZEMEK R. 2000a: Wing movement behavior in longand short- winged morphs of the flightless bug Pyrrhocoris apterus L. (Heteroptera: Pyrrhocoridae). J. Insect Behav. 13: 741-750.

SOCHA R. \& ZEMEK R. 2000b: Locomotor activity in adult Pyrrhocoris apterus (Heteroptera) in relation to sex, physiological status and wing dimorphism. Physiol. Entomol. 25: 383-389. 
Socha R., Kodrík D. \& ZeMEK R. 1999a: Adipokinetic hormone stimulates insect locomotor activity. Naturwissenschaften $\mathbf{8 8}$ $85-86$.

SOCHA R., Kodrík D. \& ZeMEK R. 1999b: Stimulation of locomotion in Pyrrhocoris apterus (Heteroptera: Pyrrhocoridae) is wing-morph independent and correlated with lipid mobilization by adipokinetic hormone. Eur. J. Entomol. 96: 459-461.

Socha R., Sula J. \& ZemeK R. 1997: Feeding, drinking an digestive enzyme activities in long- and short- day females of Pyrrhocoris apterus (Heteroptera). Physiol. Entomol. 22: 161169.

SOCHA R., Śula J. \& ZEMEK R. 1998: Feeding behaviour, digestive physiology and lipid content in macropterous females of Pyrrhocoris apterus (Heteroptera: Pyrrhocoridae). Physiol. Entomol. 22: 91-96.
ŠUla J., KodRík D. \& Socha R. 1995: Hexameric heamolymph protein related to adult diapause in the red firebug, Pyrrhocoris apterus (L.) (Heteroptera). J. Insect Physiol. 41: 793-800.

VAN MarReWIJK W.J.A., VAN DeN Broek A.T.M., VAN DER Horst D.J. \& BeEnAKKers A.M.T. 1984: Hypertrehalosaemic and hyperlipaemic responses to adipokinetic hormone in fifth larval instar locust, Locusta migratoria. Insect Biochem. 14: 151-157.

ZÖllner N. \& KIRSCH K. 1962: Über die quantitative Bestimmung von Lipoiden (Mikromethode) mittels der vielen natïrlichen Lipoiden (allen bekannten Plasmalipoiden) gemeinsamen Sulfophosphovanillin-Reaktion. Z. Gesamte Exp. Med. 135: 545-561.

Received June 8, 2001; revised September 9, 2001; accepted October 23, 2001 